PAPER REFERENCE: A-VI.3

\title{
CHANGE MOBILITY IN CONJUGATED POLYMER MOLECULES
}

\author{
A.M. Almeida, ${ }^{*}$ M.M.D. Ramos, H.G. Correia \\ Departamento de Física, Universidade do Minho, Largo do Paço, \\ 4700-320 Braga, Portugal
}

\begin{abstract}
The use of conjugated polymers as active media in organic semiconductor devices demands a deep knowledge of the electronic processes involved at molecular level. Here we report the results obtained from self-consistent molecular dynamics calculations at the CNDO (Complete Neglect of Differential Overlap) level concerning intra-chain charge mobility for two well known polymers: polydiacetylene (PDA) and poly(p-phenylene vinylene) (PPV).
\end{abstract}

\footnotetext{
* Corresponding Author: Tel:+351 253 604062; Fax:+351 253678 981; E-mail: coimbra@fisica.uminho.pt
} 
Keywords: PPV, PDA, charge mobility, polaron.

\section{Introduction}

The growing interest in organic semiconductor based devices together with the most recent computational facilities have allowed the extensive use of simulations to study large molecular systems anticipating some of their individual physical properties.

For those purposes, conjugated polymers play a major role, in which their delocalised $\pi$ molecular orbitals are responsible for their special electrical and optical properties. The most used polymers are polydiacetylene (PDA) and poly(p-phenylene vinylene) (PPV) and computer simulations may provide a fundamental understanding of the electronic processes at molecular level.

\section{Method and objects}

\subsection{The theoretical method}

The approach we adopt combines a self-consistent quantum mechanical method and molecular dynamics. We have used an approximate molecular orbital method, called CNDO (Complete Neglect of Differential Overlap) within a minimal-basis representation of the valence electron wavefunctions considered in the LCAO (Linear Combination of Atomic Orbital) approximation. The gradients of the total self- 
consistent energy, evaluated at the given atom positions, yield the interatomic forces, which will be used in a molecular dynamical method to move the atoms. The atoms of an initial structural configuration move, within the Born-Oppenheimer approximation, on the potential energy surface obtained directly from the electronic structure calculation.

\subsection{The molecules to study}

The molecules considered in this paper are PDA and PPV strands with 16 repeated units. They are kept in the ground state. When charge is injected, the spin multiplicity is chosen to correspond to the lowest energy configuration.

\section{The injection of charges in the molecules}

\subsection{The dimerisation parameters}

Simulations of uncharged PPV and PDA molecules were carried out in order to obtain their relaxed geometries, i. e., the spatial arrangement of the molecules atoms which minimises the clusters energy. Then, the molecules were charged by the addition or removal of electrons, and new relaxed geometries were obtained.

The comparison between uncharged and charged polymer backbones of each kind is done upon the evaluation of the bond length differences as seen at each carbon site relative to its neighbour carbon atoms. Usually, those differences are called the dimerisation parameters. 
From the observation of the dimerisation parameters it is identified one structural defect for each injected charge. For a single injected charge, the defect is localised at the centre of the molecule; two charges induce two defects close to the ends of the chain. Figure 1 shows the changes on the dimerisation parameters for a triple charged PDA strand (squares for holes, circles for electrons). In this case, there are three induced defects whose localisation and shape are independent of the injected charge sign.

\subsection{The Mulliken charge distribution}

From the simulations results, it is also possible to retrieve the valence electron density. The net charge for each atom, calculated by the difference in its valence electron density in the molecule from the value for the isolated atom, is evaluated, thus defining the Mulliken atomic charge. The comparison of the Mulliken charges for all the atoms between charged and uncharged molecules makes it possible to map the distribution of the injected charges in the molecules.

As can be seen from Figure 2, the number of injected charges is equal to the number of delimited regions where the electron density is modified.

For practical purposes, another way to look at the charge variation is to plot the Mulliken charge associated to each atomic species ( $\mathrm{H}$ or $\mathrm{C}$ ) against the unit position along the polymer chain (see Figure 3). 


\subsection{The polarons}

Although illustrated for PDA, the dimerisation parameters and the Mulliken charge patterns show similar behaviour for PPV [1]. In both cases, the spatial superposition of the Mulliken charge patterns and the structural defects induced by the injected charges make it immediate to identify the existence of a polaron [2] associated with each injected charge.

\section{Charge carrier drift}

The effect of an external electric field can be appreciated in order to estimate the charge mobility along these molecules. The minimum value for the electric field to produce some noticeable changes in both charge and dimerisation patterns depends on the polymer chemistry [3], on the number and on the sign of the charges [4]. Two regimes for the charge mobility can be identified [4], each one corresponding to a different reaction of the polarons to the applied electric field.

\subsection{The polaron}

Under an external electric field between $2.0 \times 10^{8}$ and $2.47 \times 10^{8} \mathrm{~V} / \mathrm{m}$ (see Table 1 ), one positive net charge drifts from the centre to the end of the PPV molecule. The structural defect accompanies the charge.

If the field is higher than $2.49 \times 10^{8} \mathrm{~V} / \mathrm{m}$, the charge is already localised at the end of the polymer chain after the first time-step of the calculation. The structural defect, 
once localised at the chain centre, vanishes completely, whereas in the new charge site, a new defect emerges, as can be seen by the oscillations of the dimerisation pattern.

In the first case the field is not strong enough to decouple the polaron, so the charge moves slowly thus allowing the chain distortion to adjust almost instantaneously (at each simulation time-step) to the evolving electron density.

On the other hand, when the field is very strong, the charge is suddenly displaced to the end of the chain leaving behind a bare charge defect that will be relaxed to a defect-free structure. The new charge sites will rearrange to accommodate the arrived charge. In this way, it can be considered the initial polaron was destroyed and another one was raised.

\subsection{The bipolaron}

Two similar charges in the same molecule with their corresponding dimerisation patterns (near the chain ends) are said to form a bipolaron [5].

An external electric field gives rise to the motion of both polarons in the field direction. One of the polarons is pushed against the nearest chain end. The other one is pushed in the same direction. Depending on the field strength, the polarons may be displaced, they may not move (zero or weak field) or one of the polarons may be slightly displaced and the other is destroyed in its former site to be rebuilt in a new position (strong field). 


\section{Charge mobility}

Under moderate electric field intensities, it is possible to adopt suitable criteria to measure the length of the charge displacement and the corresponding drift of the structural defect when the polaron is not destroyed, as a function of time of both the dimerisation pattern and the Mulliken charge distribution per chain unit. The charge mobility thus calculated is of the order of $4 \mathrm{~cm}^{2} \mathrm{~V}^{-1} \mathrm{~s}^{-1}$ for PDA and $3 \mathrm{~cm}^{2} \mathrm{~V}^{-1} \mathrm{~s}^{-1}$ for PPV.

For fields of higher intensity, the polaron is decoupled, the charge being immediately displaced to the end of the chain. Then, only a rough estimate can be appointed to the charge mobility, which is of the order of $22 \mathrm{~cm}^{2} \mathrm{~V}^{-1} \mathrm{~s}^{-1}$ for PDA and over $75 \mathrm{~cm}^{2} \mathrm{~V}^{-1} \mathrm{~s}^{-1}$ for PPV.

The figures just mentioned refer only to single charged molecules, irrespective of the charge sign. Table 1 and Figure 4 illustrate the charge mobility regimes just described with their respective electric field ranges, for PPV and PDA with charges ranging from -2 to +2 electrons.

For field intensities higher than those tabulated for each case, the simulations failed to yield feasible physical results. 
Transient Current [6] and Time of Flight [7][8] measurements for PPV thin films

reported by several authors indicate charge mobility of the order of $10^{-7} \mathrm{~cm}^{2} \mathrm{~V}^{-1} \mathrm{~s}^{-1}$ [6] [7] or $10^{-4} \mathrm{~cm}^{2} \mathrm{~V}^{-1} \mathrm{~s}^{-1}$ for holes and $10^{-6} \mathrm{~cm}^{2} \mathrm{~V}^{-1} \mathrm{~s}^{-1}$ for electrons [8].

Other report refers charge carrier mobility, presumably due to the holes, close to 0.1 $\mathrm{cm}^{2} \mathrm{~V}^{-1} \mathrm{~s}^{-1}[9]$.

\section{Conclusions}

Our calculations refer to intra-chain charge mobility, so our results are not directly comparable to the experimental ones. However, we may envisage the large differences found in charge mobility as the result of the inter-chain charge hopping process, which is dependent on the polymer film properties such as molecular arrangement, chain length distribution, crosslinking, etc.

The PPV molecules are likely to respond more efficiently and require lower electric field intensity to move the charge carriers along the chain than PDA. The transition between low and high mobility regimes is sharper for PPV, making it more adequate to fine-tuning of the applied working voltage.

Additionally, it is known that long polymer strands contribute to electroluminescence resulting from charge recombination [10], unlike PDA, for which only small molecules luminesce [11]. The use of longer molecules in a device should favour the bulk electric current density because there are less hops between chains, thus providing more chances for the charges to recombine. 


\section{References}

[1] M.M.D. Ramos, A.M. Almeida, Vacuum 64 (2002) 99.

[2] A.J. Fisher, W. Hayes, D.S. Wallace, J. Phys.: Condens. Matter. 1 (1989) 5567.

[3] A.M. Almeida, M.M.D. Ramos, A.M. Cadilhe, Comp. Mater. Sci. 24 (2002) 54.

[4] A.M. Almeida, M.M.D. Ramos, R.M. Ribeiro, Macromol. Symp. 181 (2002) 479.

[5] J.H. Burroughes, D.C.C. Bradley, A.R. Brown, R.N. Marks, K. Mackay, R.H. Friend, P.L. Burns, A.B. Holmes, Nature 347 (1990) 539.

[6] E. Pinotti, A. Sassella, A. Borghesi, R. Tubino, Syn. Met. 122 (2001) 169.

[7] E. Lebedev, S. Karg, W. Brütting, Appl. Phys. Lett. 71 (1997) 2686.

[8] H.M. Lee, D.K. Oh, C.H. Lee, C.E. Lee, D.W. Lee, J.-I. Jin, Syn. Met. 119 (2001) 473.

[9] N. Tessler, N.T. Harrison, R.H. Friend, Adv Mater. 10 (1998) 64.

[10] M. Wohlgenannt, X.M. Jiang, Z.V. Vardeny, R.A.J. Janssen, Phys. Rev. Lett. 88 (2002) 197401.

[11] H. Sixl, in: D. Bloor (Ed.), Polydiacetilene, Nijhoff, Amsterdam, 1984, pp 240245. 
PAPER REFERENCE: A-VI.3

\begin{tabular}{ccccccc} 
& & \multicolumn{4}{c}{ Electric field intensity $\left(\mathrm{x} 10^{8} \mathrm{Vm}^{-1}\right)$} \\
\cline { 3 - 4 } \cline { 6 - 7 } Polymer & & $\mathrm{Q}=-2$ & $\mathrm{Q}=-1$ & $\mathrm{Q}=+1$ & $\mathrm{Q}=+2$ \\
\multirow{2}{*}{ PPV } & Low mobility & {$[2.2-3.0]$} & {$[1.55-2.60]$} & & {$[2.00-2.47]$} & {$[2.2-3.25]$} \\
& High mobility & {$[3.15-9.75]$} & {$[2.70-11.00]$} & & {$[2.49-11.00]$} & {$[3.3-9.75]$} \\
\hline \multirow{2}{*}{ PDA } & Low mobility & {$[2.02-6.50]$} & {$[2.347-5.08]$} & & {$[1.60-4.70]$} & {$[2.62-6.50]$} \\
& High mobility & {$[7.00-12.50]$} & {$[5.12-14.70]$} & & {$[5.10-14.90]$} & {$[6.75-13.00]$} \\
\hline
\end{tabular}

Table 1 - Electric field ranges for low and high intra-molecular charge mobility in PPV and PDA chains with 16 repeated units as a function of the cluster net charge Q. 
List of figure captions:

Figure 1. Variation of the dimerisation parameters for a triple charged 16-monomer PDA chain.

Figure 2. Variation of the Mulliken charge for each carbon atom when a 16-monomer PDA chain has a net charge of 3 electrons.

Figure 3. Variation of the Mulliken charge per atom type for each unit of a PPV strand with a total net charge of 2 electrons.

Figure 4. Charge carrier mobility regimes for single and doubly charged PPV and PDA strands. 
PAPER REFERENCE: A-VI.3

Figure 1.

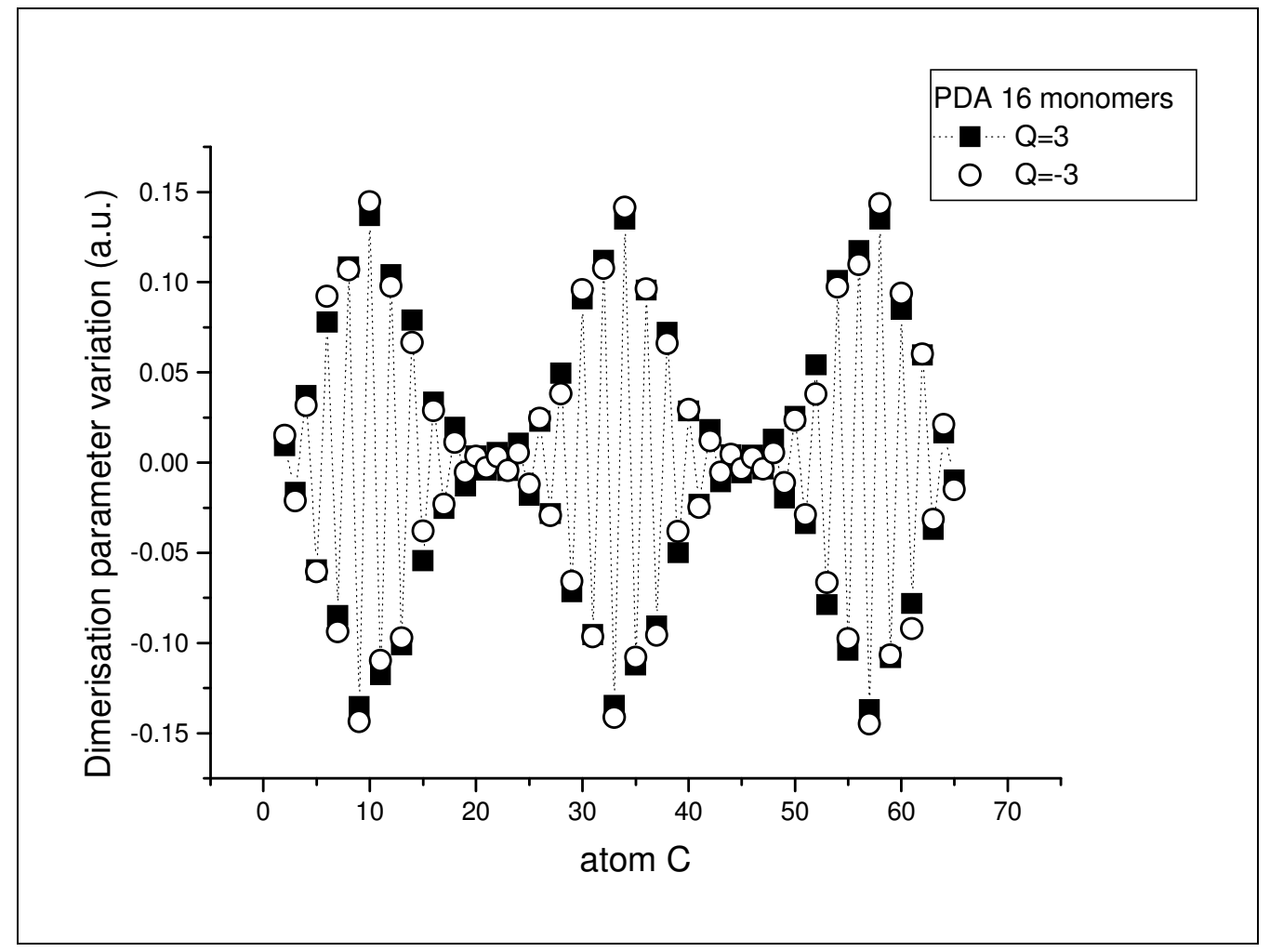


Figure 2.

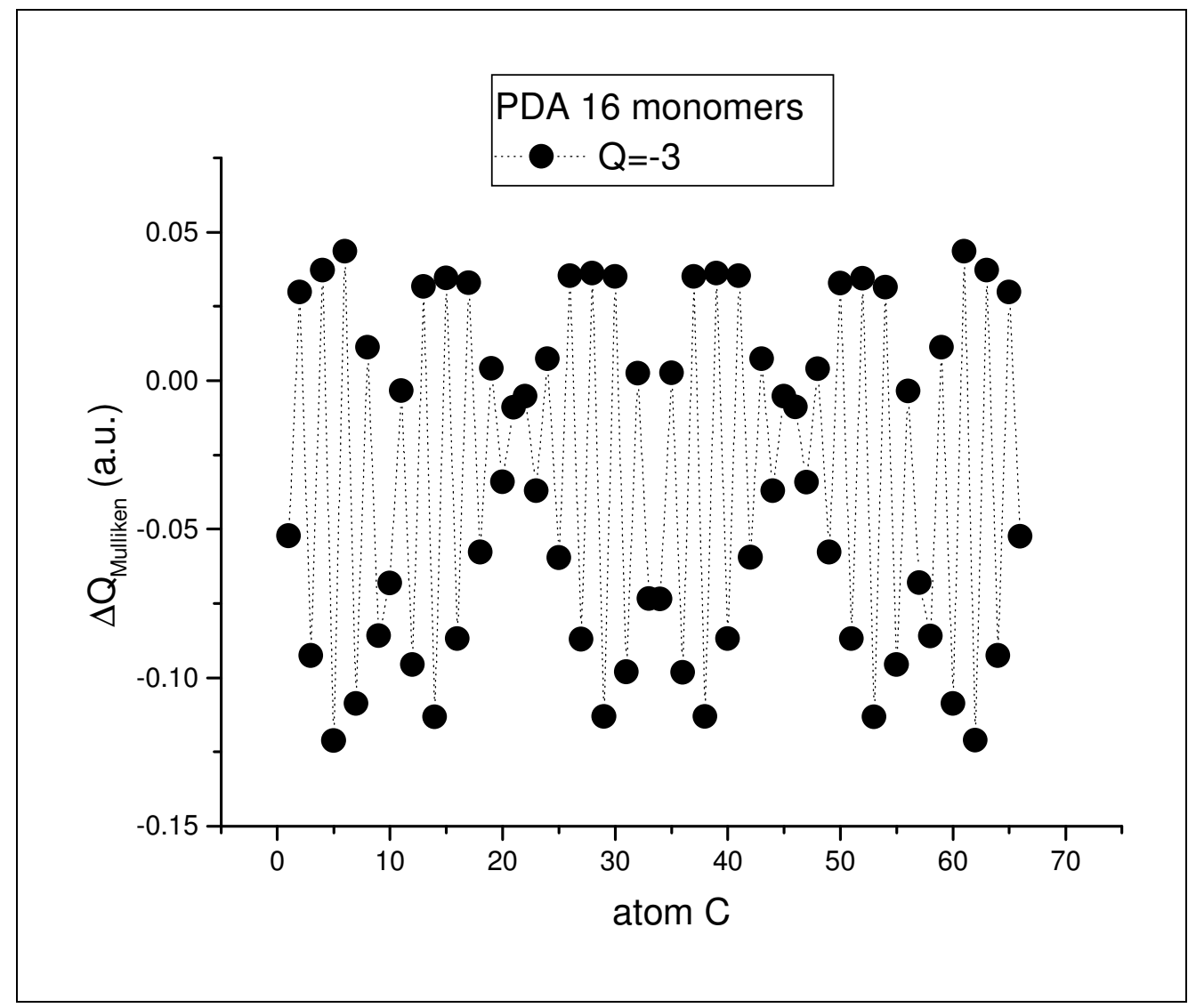


PAPER REFERENCE: A-VI.3

Figure 3.

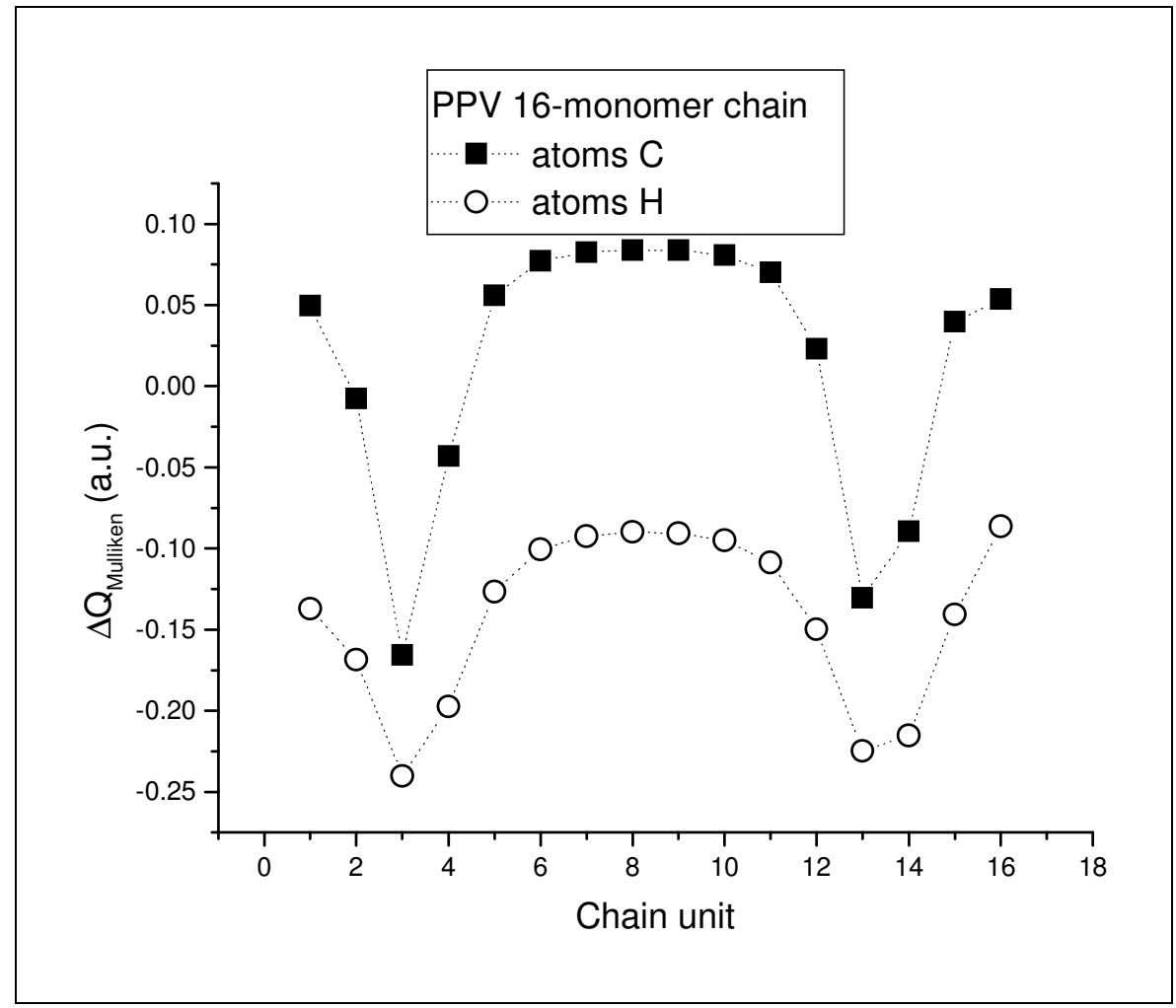


PAPER REFERENCE: A-VI.3

Figure 4.

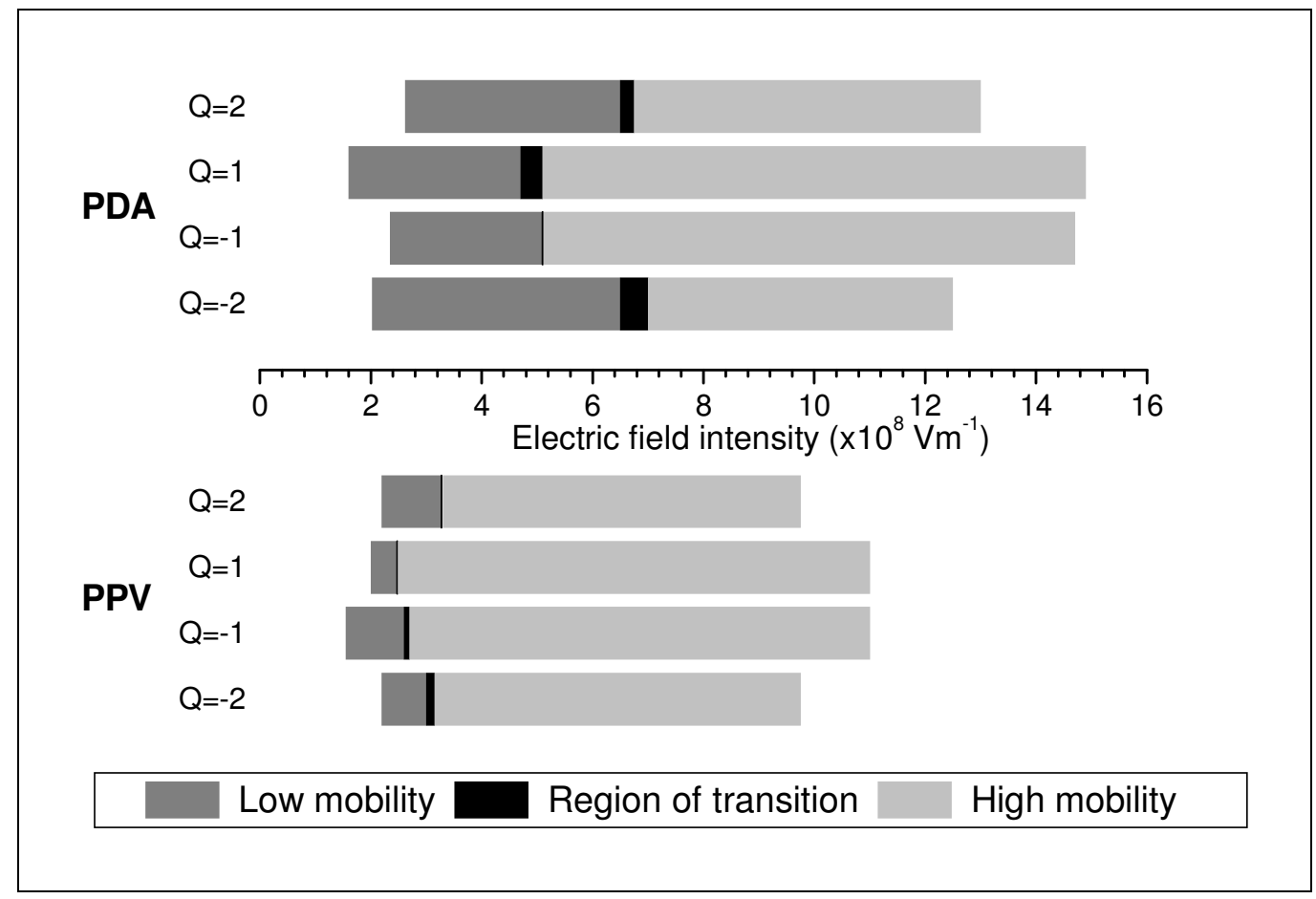

